\title{
Our Lyon Brace with removable neck ring (preliminary study) B Iemolo
}

Address: Institution: I.S.C. (Italian Spine Center) Via dei Mille n. 238 Vittoria 97019 (Rg), Italy

Email: B Iemolo - biagioiemolo@alice.it

from 6th International Conference on Conservative Management of Spinal Deformities Lyon, France. 2I-23 May 2009

Published: I4 December 2009

Scoliosis 2009, 4(Suppl 2):O47 doi:I0.1 186/1748-7|6I-4-S2-O47

This abstract is available from: http://www.scoliosisjournal.com/content/4/S2/O47

(c) 2009 lemolo; licensee BioMed Central Ltd.

\section{Objectives}

The purpose of this study is to present a spinal orthosis to treat High Apex Thoraco-Lumbar Scoliosis (HA-TLS) according to biomechanical criteria. Our orthosis is a Lyon brace modified by a removable elongation neck ring which is easily usable during nighttime, and which maximizes compliance during full-time brace treatment with a brace not visible during the daytime.

\section{Background}

HA-TLS is very difficult to correct using other braces except for the Milwaukee brace (MB). Since the introduction of the $\mathrm{MB}$, a major problem has been poor patient compliance due to the perception of the brace as cosmetically unacceptable. The forces applied to the spine by the MB are well illustrated by White-Panjabi (1). According to circadian biological rhythm, a sleeping person R.E.M. has decreased muscular tone, and gravitational loading force on the horizontal spine is absent. Therefore, common sense tells us to strike scoliosis at night with two combined forces: torsional forces provided by the Lyon brace plus very important elongation forces provided by the neck ring.

\section{Methods and results}

Inclusion criteria were as follows: girls, idiopathic scoliosis with apex curves cephalic to T8, growing age (10-14 years), Cobb angle of minimum $25^{\circ}$ and maximum $45^{\circ}$. The Risser sign value was less than 3 . The group consisted of 32 girls wearing our brace for more than 3 months, with a minimum time of wearing of 21 hours per day and using the neck ring at night. The braces were all made in the same workshop, and the treatment was managed by the same physician (2).

\section{Outcome}

The authors' follow-up is too small (interim results). However, radiographic control in the brace after 3 months (usually required in our protocol) demonstrated excellent initial correction of HA-TLS in all patients. The average initial in-brace correction was $45 \%$ for major curves of $35^{\circ}$ and $65 \%$ for minor curves.

\section{Conclusion}

HA-TLS represents a significant challenge for the physician. The neck ring makes the MB brace cosmetically unacceptable to many patients. Bracing is useless without compliance (3). During growth, we believe that the psychological factor is very important, helping to obtain early improvement of compliance and a positive body image in all patients. Our orthosis has a very minor effect on the quality of life and represents an alternative brace to treat HA-TLS in adolescents. 